\title{
Canonical Commutator and Mass Renormalization
}

\author{
G. W. Ford ${ }^{1}$ and R. F. O'Connell ${ }^{2}$
}

Received February 6, 1989

The question considered is the effect on the canonical commutation rules of mass renormalization in quantum electrodynamics. This is investigated by considering the quantum Langevin equation for a charged quantum oscillator interacting with the radiation field via dipole interaction. Explicit expressions for the commutator are obtained for finite cutoff frequency as well as the large-cutoff limit. The surprising result obtained is that the exactly equal-time (canonical) commutator involves the bare mass, while the corresponding commutator for infinitesimally displaced times involves the renormalized mass.

KEY WORDS: Canonical commutator; Langevin equation; quantum electrodynamics; commutation relation.

An integral feature of elementary quantum mechanics is the canonical coordinate momentum commutation relation:

$$
[x(t), p(t)]=i \hbar
$$

For our purposes we use the fact that for a nonrelativistic particle $p=m \dot{x}$, where $m$ is the particle mass and a dot denotes the time derivative, to write this in the form

$$
[x(t), \dot{x}(t)]=i \hbar / m
$$

Here, lest we confuse the reader, we remark that for a particle interacting with the electromagnetic field in the often-used Coulomb gauge the canonical momentum is $p=m \dot{x}+(e / c) A_{x}(x, t)$, where $A_{x}$ is the $x$ component of the vector potential operator. Since $x$ commutes with $A_{x}$, we get the same result (2).

\footnotetext{
${ }^{1}$ Department of Physics, University of Michigan, Ann Arbor, Michigan 48109-1120.

${ }^{2}$ Department of Physics and Astronomy, Louisiana State University, Baton Rouge, Louisiana 70803-4001.
} 
The purpose of this paper is to address the question of the effect of mass renormalization on this relation. In particular, we ask: Is the mass in (2) the renormalized mass? Surprisingly, to our knowledge this particular question has not been addressed in the literature. Our approach to this question is to analyze the exactly solvable system of a charged quantum oscillator interacting with the radiation field via dipole interaction. The result we find is that the mass in (2) is the bare mass, but that the renormalized mass occurs in the corresponding commutator in which the two times are not exactly equal, but infinitesimally separated.

The framework within which we analyze this system is the quantum Langevin equation

$$
m \ddot{x}+\int_{-\infty}^{t} d t^{\prime} \mu\left(t-t^{\prime}\right) \dot{x}\left(t^{\prime}\right)+K x=F(t)
$$

where $K$ is the oscillator force constant and $m$ is the bare mass. This is a Heisenberg equation of motion for the coordinate operator $x$. The coupling with the heat bath is described by two terms: an operator-valued random force $F(t)$ with mean zero, and a mean force characterized by the memory function $\mu(t)$. The (symmetric) autocorrelation of $F(t)$ is

$\frac{1}{2}\left\langle F(t) F\left(t^{\prime}\right)+F\left(t^{\prime}\right) F(t)\right\rangle$

$$
=\frac{1}{\pi} \int_{0}^{\infty} d \omega \operatorname{Re}\left\{\tilde{\mu}\left(\omega+i 0^{+}\right)\right\} \hbar \omega \operatorname{coth}\left(\frac{\hbar \omega}{2 k T}\right) \cos \omega\left(t-t^{\prime}\right)
$$

and the nonequal-time commutator of $F(t)$ is

$$
\left[F(t), F\left(t^{\prime}\right)\right]=\frac{2}{i \pi} \int_{0}^{\infty} d \omega \operatorname{Re}\left\{\tilde{\mu}\left(\omega+i 0^{+}\right)\right\} \hbar \omega \sin \omega\left(t-t^{\prime}\right)
$$

In these expressions

$$
\tilde{\mu}(\omega)=\int_{0}^{\infty} d t \mu(t) e^{i \omega t}, \quad \operatorname{Im} \omega \geqslant 0
$$

is the Fourier transform of the memory function. This description in terms of the quantum Langevin equation and the accompanying properties of the operator random force are discussed in some detail in a recent publication. ${ }^{(1)}$ In particular, the description is uniquely determined by such general principles as the existence of a ground (equilibrium) state of the system and causality. More particularly, $\tilde{\mu}(\omega)$ must be a positive real function, analytic in the upper half-plane, with real part positive, and satisfying the reality condition $\tilde{\mu}(\omega)^{*}=\tilde{\mu}(-\omega)$. Positive real functions have 
many remarkable properties, but for our purposes we note only that, for $|\omega| \rightarrow \infty$ in the upper half-plane, $\tilde{\mu}(\omega)$ must have the asymptotic form

$$
\tilde{\mu}(\omega) \approx-i c_{1} \omega+c_{2}+i c_{3} / \omega
$$

where $c_{1}, c_{2}$, and $c_{3}$ are positive constants at least one of which must be nonzero.

We first obtain a general expression for the nonequal-time commutator of $x(t)$ based on this description. The solution of the quantum Langevin equation can be written as

$$
x(t)=\int_{-\infty}^{\infty} d t^{\prime} G\left(t-t^{\prime}\right) F\left(t^{\prime}\right)
$$

where $G(t)$ is the (retarded) Green function. The Fourier transform of $G(t)$ is the polarizability

$$
\alpha(\omega)=\int_{0}^{\infty} d t G(t) e^{i \omega t}, \quad \operatorname{Im} \omega \geqslant 0
$$

where we have used the fact that the retarded Green function vanishes for negative times. From (3) we find

$$
\alpha(\omega)=\left[-m \omega^{2}+K-i \omega \tilde{\mu}(\omega)\right]^{-1}
$$

Forming the nonequal-time commutator of $x(t)$ with this solution, we get

$$
\begin{aligned}
{\left[x(t), x\left(t^{\prime}\right)\right]=} & \int_{-\infty}^{\infty} d t_{1} \int_{-\infty}^{\infty} d t_{1}^{\prime} G\left(t-t_{1}\right) \\
& \times G\left(t^{\prime}-t_{1}^{\prime}\right)\left[F\left(t_{1}\right), F\left(t_{1}^{\prime}\right)\right]
\end{aligned}
$$

Inserting the expression (5) for the commutator $F(t)$, we find

$$
\begin{aligned}
{\left[x(t), x\left(t^{\prime}\right)\right]=} & \frac{2 \hbar}{i \pi} \int_{0}^{\infty} d \omega \omega|\alpha(\omega)|^{2} \\
& \times \operatorname{Re}\{\tilde{\mu}(\omega)\} \sin \omega\left(t-t^{\prime}\right)
\end{aligned}
$$

where we have used (9) and the reality condition $\alpha(-\omega)=\alpha(\omega)^{*}$. But from (10) it is clear that

$$
\omega|\alpha(\omega)|^{2} \operatorname{Re}\{\tilde{\mu}(\omega)\}=\operatorname{Im}\{\alpha(\omega)\}
$$

so that (12) may be written in the form

$$
\left[x(t), x\left(t^{\prime}\right)\right]=\frac{2 \hbar}{i \pi} \int_{0}^{\infty} d \omega \operatorname{Im}\{\alpha(\omega)\} \sin \omega\left(t-t^{\prime}\right)
$$


This is the general expression for the commutator we seek. An equivalent result was obtained by Lewis and Thomas. ${ }^{(2)}$ Note that the commutator is a $c$-number. [We remark that if we were to form the expectation of this expression, we would obtain a result familiar in discussions of the fluctuation-dissipation, theorem see ref. 3, Eq. (2.15).] If now we form the derivative of both sides of (14) with respect to $t^{\prime}$, we get the equivalent result

$$
\left[x(t), \dot{x}\left(t^{\prime}\right)\right]=\frac{2 i \hbar}{\pi} \int_{0}^{\infty} d \omega \omega \operatorname{Im}\{\alpha(\omega)\} \cos \omega\left(t-t^{\prime}\right)
$$

This formula will be the basis for our further discussion.

Before proceeding to the electrodynamic case, it will be instructive to consider the case of constant friction, for which $\tilde{\mu}(\omega)=\zeta$, the friction constant, and (10) takes the form

$$
\begin{aligned}
\alpha(\omega) & =\left(-m \omega^{2}+K-i \omega \zeta\right)^{-1} \\
& \equiv m^{-1}\left(-\omega^{2}+\omega_{0}^{2}-i \omega \gamma\right)^{-1}
\end{aligned}
$$

Putting this in (15), one can perform the integral by contour methods to give

$$
\begin{aligned}
{\left[x(t), \dot{x}\left(t^{\prime}\right)\right]=} & i \frac{\hbar}{m}\left[\cos \omega_{1}\left(t-t^{\prime}\right)\right. \\
& \left.-\frac{\gamma}{2 \omega_{1}} \sin \omega_{1}\left|t-t^{\prime}\right|\right] \exp \left(-\frac{\gamma}{2}\left|t-t^{\prime}\right|\right)
\end{aligned}
$$

where

$$
\omega_{1}=\left(\omega_{0}^{2}-\gamma^{2} / 4\right)^{1 / 2}
$$

For $t^{\prime}=t$, we get the canonical commutator (2) with $m$ the particle mass. This result for the equal-time commutator in the constant friction case was recently obtained by Dekker ${ }^{(4)}$ and still earlier by Milonni. ${ }^{(5)}$ We emphasize that for this constant-friction case the bare mass $m$ is also in fact the observed (physical) mass, so that no question of mass renormalization arises.

This result for the canonical equal-time commutator is general, requiring only that for $\omega$ large the mass term dominates the asymptotic form of the polarizability. Stated more precisely, the requirement is that the constant $c_{1}$ in the general asymptotic form (7) of $\tilde{\mu}(\omega)$ must be zero. (An example of an exception is a Stokes sphere moving in a viscous fluid. ${ }^{(6)}$ ) To 
demonstrate this general result, we first put $t^{\prime}=t$ in (15) and then use the reality condition, which requires that the real and imaginary parts of $\alpha(\omega)$ be, respectively, even and odd functions of $\omega$, to write

$$
[x(t), \dot{x}(t)]=\frac{\hbar}{\pi} \int_{-\infty}^{\infty} d \omega \omega \alpha(\omega)
$$

We now use the fact that $-i \omega \alpha(\omega)$ is a positive real function with no singularities in the upper half-plane. ${ }^{(1)}$ The path of integration can therefore be deformed upward into a large semicircle, $\omega=R e^{i \phi}$. On this semicircle, assuming only that the constant $c_{1}$ in the asymptotic form (7) of $\mu(\omega)$ vanishes, we see from $(10)$ that in general $\alpha(\omega) \approx-1 / m \omega^{2}=$ $-\left(1 / m R^{2}\right) e^{-2 i \phi}$, and (19) becomes

$$
[x(t), \dot{x}(t)]=-i \frac{\hbar}{m \pi} \int_{\pi}^{0} d \phi=\frac{i \hbar}{m}
$$

Thus we see that for any "normal" case, for which the constant $c_{1}$ in (7) is zero and $\tilde{\mu}(\omega)$ remains bounded as $\omega \rightarrow \infty$, the canonical commutation relation (2) holds with $m$ the particle mass.

We now consider the electrodynamic case. Here, the function $\tilde{\mu}(\omega)$ can be expressed in the form ${ }^{(1,7)}$

$$
\tilde{\mu}(\omega)=2 e^{2} \Omega^{2} \omega / 3 c^{3}(\omega+i \Omega)
$$

where $\Omega$ is a larger cutoff frequency that characterizes the electron form factor. The corresponding expression for the polarizability is

$$
\alpha(\omega)=\frac{\omega+i \Omega}{-m \omega^{3}-i \Omega\left(m+2 e^{2} \Omega / 3 c^{3}\right) \omega^{2}+K \omega+i \Omega K}
$$

The first thing we note here is that, for any fixed value of the cutoff frequency $\Omega$, this electrodynamic case is a "normal" case; the function $\tilde{\mu}(\omega)$ given by (21) has the asymptotic form (7) with $c_{1}=0$. As we have just shown, this means that the canonical equal-time commutation relation (2) holds. But now $m$ is the bare mass.

The renormalized mass appears when we consider the nonequal-time commutator for time differences which are not unphysically short. In the expression (15) for the commutator this means that we look at a scale of frequencies that are physically high, i.e., high compared with the natural frequency of the oscillator, yet small compared with the cutoff frequency $\Omega$. On this scale $\tilde{\mu}(\omega) \cong-i 2 e^{2} \omega \Omega / 3 c^{3}$, and the polarizability (22) takes the form

$$
\alpha(\omega) \cong-1 / M \omega^{2}
$$


where $M$ is the renormalized mass:

$$
M=m+2 e^{2} \Omega / 3 c^{3}
$$

The renormalized mass is identified as the observed mass; the bare mass appears only when the system is probed with frequencies of the order of $\Omega$. We can take this into account if we factor the denominator in (22) to write

$$
\alpha(\omega)=\frac{1}{m} \frac{\omega+i \Omega}{\left(\omega+i \Omega^{\prime}\right)\left(-\omega^{2}+\omega_{0}^{2}-i \omega \gamma\right)}
$$

where we have expressed the parameters $K, M$, and $\Omega$ in terms of parameters $\omega_{0}, \gamma$, and $\Omega^{\prime}$ through the relations

$$
\frac{1}{\Omega}=\frac{1}{\Omega^{\prime}}+\frac{\gamma}{\omega_{0}^{2}}, \quad \frac{K}{M}=\frac{\omega_{0}^{2} \Omega^{\prime}}{\Omega^{\prime}+\gamma}, \quad \frac{M}{m}=\frac{\left(\omega_{0}^{2}+\gamma \Omega^{\prime}\right)\left(\Omega^{\prime}+\gamma\right)}{\omega_{0}^{2} \Omega^{\prime}}
$$

Putting the form (25) in the general expression (15), we can perform the integral by contour methods to get

$$
\begin{aligned}
{\left[x(t), \dot{x}\left(t^{\prime}\right)\right]=} & \frac{i \hbar \Omega^{\prime}\left(\Omega^{\prime}+\gamma\right)}{M \omega_{0}^{2}\left(\Omega^{\prime 2}+\omega_{0}^{2}-\gamma \Omega^{\prime}\right)}\left\{\left[\frac{\Omega^{\prime 2}\left(\omega_{0}^{2}-\gamma^{2}\right)+\omega_{0}^{4}}{\Omega^{\prime 2}}\right.\right. \\
& \times \cos \omega_{1}\left(t-t^{\prime}\right)-\frac{\gamma\left[\Omega^{\prime 2}\left(3 \omega_{0}^{2}-\gamma^{2}\right)+\omega_{0}^{4}\right]}{2 \omega_{1} \Omega^{\prime 2}} \\
& \left.\times \sin \omega_{1}\left|t-t^{\prime}\right|\right] \exp \left(-\frac{\gamma}{2}\left|t-t^{\prime}\right|\right) \\
& \left.+\gamma \Omega^{\prime} \exp \left(-\Omega^{\prime}\left|t-t^{\prime}\right|\right)\right\}
\end{aligned}
$$

If we set $t^{\prime}=t$ and use the expression (26) for the renormalized mass, we obtain exactly the canonical commutator (2) with $m$ the bare mass, as we now know on general grounds we must.

We now consider the large-cutoff limit. In doing this we must recognize that the relation (24) between the bare and renormalized masses is in fact a constraint on $\Omega$. The point is that $M$ must be fixed at the physically observed mass and, therefore, if the cutoff frequency is taken to be too large, the bare mass will become negative, which is unacceptable on general physical grounds. The largest possible cutoff corresponds therefore to the bare mass equal to zero, which from (24) we see corresponds to

$$
\Omega=1 / \tau_{e}, \quad \tau_{e}=2 e^{2} / 3 M c^{3} \cong 6.24 \times 10^{-24} \mathrm{sec}
$$

The time $\tau_{e}$, which is $2 / 3$ the time required for a photon to traverse the classical electron radius, is very short, far shorter than any of the natural 
times of a nonrelativistic system. The frequency $\Omega$ is correspondingly large. For example, the cutoff frequency occurring in the nonrelativistic calculation of the Lamb shift is of the order of $M c^{2} / \hbar{ }^{(8)}$ while that given in (28) is $\hbar c / e^{2}=137$ times larger. Thus, although $\Omega$ is in fact finite, it is sufficiently large that it is effectively infinite.

The cutoff (28) corresponds to the parameter $\Omega^{\prime}$ in (26), what we might call the renormalized cutoff, going to infinity. In this limit it is a simple matter to show

$$
K=M \omega_{0}^{2}, \quad \gamma=\omega_{0}^{2} \tau_{e}, \quad \Omega^{\prime} \rightarrow \infty
$$

and in this same limit the polarizability (25) becomes

$$
\alpha(\omega)=\left(\frac{-M \omega^{2}}{1-i \omega \tau_{e}}+K\right)^{-1}=\frac{1}{M} \frac{1-i \omega \tau_{e}}{-\omega^{2}+\omega_{0}^{2}-i \omega \gamma}
$$

This is not a "normal" polarizability! The asymptotic form for large $\omega$ is $\alpha(\omega) \approx i \tau_{e} / M \omega$, not the form (23) of the "normal" case. On the other hand, this is a simple form for which the expression (15) for the commutator can be easily evaluated. We find

$$
\begin{aligned}
{\left[x(t), \dot{x}\left(t^{\prime}\right)\right]=} & \frac{i \hbar}{M}\left\{\frac{2 \gamma}{\omega_{0}^{2}} \delta\left(t-t^{\prime}\right)+\left[\left(1-\frac{\gamma^{2}}{\omega_{0}^{2}}\right) \cos \omega_{1}\left(t-t^{\prime}\right)\right.\right. \\
& \left.-\frac{\gamma\left(3-\gamma^{2} / \omega_{0}^{2}\right)}{2 \omega_{1}} \sin \omega_{1}\left|t-t^{\prime}\right|\right] \\
& \left.\times \exp \left(-\frac{\gamma}{2}\left|t-t^{\prime}\right|\right)\right\}
\end{aligned}
$$

where $\omega_{1}$ is again given by (18). The limit $\Omega^{\prime} \rightarrow \infty$ of the expression (27) gives exactly this same result, so the large-cutoff limit commutes with the evaluation of the integral in the expression (15) for the commutator. If we set $t^{\prime}=t$ in (31), we get

$$
[x(t), \dot{x}(t)]=\frac{i \hbar}{M}\left[\frac{2 \gamma}{\omega_{0}^{2}} \delta(0)+1-\frac{\gamma^{2}}{\omega_{0}^{2}}\right]
$$

This divergent expression in fact makes physical sense, since, as we have seen, the canonical equal-time commutator has the form (2) with $m$ the bare mass and in the large-cutoff limit the bare mass is zero. If, on the other hand, we take $t^{\prime} \neq t$ and then form the limit as $t^{\prime} \rightarrow t$ either from above or below, we find

$$
\left[x(t), \dot{x}\left(t+0^{ \pm}\right)\right]=(i \hbar / M)\left(1-\gamma^{2} / \omega_{0}^{2}\right)
$$


This is a finite result and corresponds to the canonical commutator (2) with the renormalized mass and a very small correction.

The quantity $\gamma^{2} / \omega_{0}^{2}=\left(\omega_{0} \tau_{e}\right)^{2}$ is indeed very small. For example, if we were to choose $\omega_{0}$ to be the Rydberg frequency, then $\gamma^{2} / \omega_{0}^{2}=\left(e^{2} / \hbar c\right)^{6} / 9 \cong$ $2 \times 10^{-14}$. This is surely negligibly small. What is more, this correction reflects the particular form we have chosen for the electron form factor, ${ }^{(1,7)}$ and has no physical significance within the nonrelativistic approximation that is the basis of our discussion. We therefore drop such corrections.

In conclusion, therefore, we see that in the nonrelativistic case the canonical commutation rule (2) strictly holds true with $m$ the bare mass. On the other hand, the renormalized mass $M$ appears in the nonequal-time commutation rule, which takes the form

$$
\begin{aligned}
{\left[x(t), \dot{x}\left(t^{\prime}\right)\right]=} & \frac{i \hbar}{M}\left\{\frac{2 \gamma}{\omega_{0}^{2}} \delta\left(t-t^{\prime}\right)+\left[\cos \omega_{1}\left(t-t^{\prime}\right)\right.\right. \\
& \left.\left.-\frac{3 \gamma}{2 \omega_{1}} \sin \omega_{1}\left|t-t^{\prime}\right|\right] \exp \left(-\frac{\gamma}{2}\left|t-t^{\prime}\right|\right)\right\}
\end{aligned}
$$

The (unphysical) bare mass has disappeared from this expression. However, the canonical commutation rule has disappeared as well and cannot be recovered except in the singular form (32). This surprising result does not seem to have been noted before.

\section{ACKNOWLEDGMENTS}

It is with pleasure that we express our admiration for the career of E. G. D. Cohen. His high standards of scholarship serve as an example for us all.

This research was partially supported by the U.S. Office of Naval Research under contract N00014-86-K-0002 and by the National Science Foundation under grant INT-8504402.

\section{REFERENCES}

1. G. W. Ford, J. T. Lewis, and R. F. O'Connell, Phys. Rev. A 37:4419 (1988).

2. J. T. Lewis and L. C. Thomas, Ann. Inst. Henri Poincaré 22:241 (1975).

3. H. B. Callen and T. A. Welton, Phys. Rev. 83:34 (1951).

4. H. Dekker, Phys. Lett. A 119:201 (1986).

5. P. W. Milonni, Phys. Lett. A 82:225 (1980).

6. L. D. Landau and E. M. Lifshitz, Fluid Mechanics (Pergamon Press, London, 1959), p. 24, Problem 5.

7. G. W. Ford, J. T. Lewis, and R. F. O'Connell, Phys, Rev. Lett. 55:2273 (1985).

8. H. A. Bethe, Phys. Rev. 72:339 (1947). 